\title{
Identifying Racial Disparities in Primary Biliary Cholangitis Patients: A Step Toward Achieving Equitable Outcomes Among All
}

\author{
A. Palermo ${ }^{1,2} \cdot$ A. Gerussi ${ }^{1,2} \cdot$ G. Mulinacci ${ }^{1,2} \cdot P$. Invernizzi $^{1,2} \cdot$ M. Carbone ${ }^{1,2}$
}

Published online: 13 August 2020

(c) Springer Science+Business Media, LLC, part of Springer Nature 2020

Primary biliary cholangitis (PBC) is a chronic liver condition characterized by a mostly indolent disease course largely managed in the outpatient setting. Hospitalizations for complications are limited to those with advanced liver disease. The prevalence and incidence of PBC appear to be increasing worldwide; however, the rates and the temporal trends of hospitalization for PBC are still unclear.

In this issue of Digestive Diseases and Sciences, Adejumo et al. [1] estimate the number of hospitalizations due to PBC between 2007 and 2014 using data from the National Inpatient Sample (NIS). Administrative databases such as the NIS have the strength to represent an entire population, overcoming limitations of single hospital-based case-finding strategies. The authors reported an increase in the number of PBC hospitalizations from 2007 to 2014. The explanations may be different from the primary assumption that the disease is becoming more virulent over time. It may rather reflect PBC patients living longer and therefore suffering from unrelated age-related morbidities; it can also be due to an increase in the number of diagnoses following improved testing and increased awareness of the condition among primary health providers.

A core finding of the work regards racial and sex differences in hospitalizations. The authors reported a lower PBC hospitalization rate among Blacks together with a higher risk of all-cause mortality. The hospitalization rate could be indirect evidence of lower access to health care, possibly due to sub-optimal outpatient management and also based on previous reports that Blacks have decreased access to therapy with ursodeoxycholic acid (UDCA; ursodiol) [2].

M. Carbone

marco.carbone@unimib.it

1 Division of Gastroenterology, Department of Medicine and Surgery, Center for Autoimmune Liver Diseases, University of Milan Bicocca, 20900 Milan, Italy

2 European Reference Network on Hepatological Diseases (ERN RARE-LIVER), San Gerardo Hospital, Monza, Italy
These pieces of evidence together with a more aggressive clinical phenotype, as supported by the higher comorbidity burden and younger age at diagnosis among Blacks, might justify the increased mortality rates in this population. This is further supported by the study of De Boer et al., who have recently shown that in the UK, Black patients with autoimmune hepatitis have a more aggressive phenotype (younger age, higher IgG levels) and have a greater risk of liver transplantation and liver-related mortality [3].

Unlike Blacks, Hispanics had a higher hospitalization rate for PBC, more often for decompensated cirrhosis compared with Whites, consistent with prior findings [4]. One possible explanation of this finding is the decreased use of primary care services among the Hispanic community. Yet, for unclear reasons, this did not translate into inferior outcomes.

Another finding of the study regards the liver biopsy rates that were higher among Blacks and Asians. This might reflect racial differences in the prevalence of AMA-negative PBC. Indeed, some studies have reported higher rates of AMA-negative disease among Asians [5], but such studies are lacking in Blacks.

As concerns sex differences, males were younger than females at the time of diagnosis (median age 58.6 vs. 62.7 years), with a larger proportion of males below 46 years and a higher frequency of comorbidities. The authors relate this to a more aggressive phenotype in males, which is in contrast to data reporting that male patients are initially evaluated at a later age and at a more advanced disease stage $[6,7]$. This discrepancy may be due to the limitations of administrative data capture based solely upon a single ICD-9 code for "biliary cirrhosis" being present. While it is likely that the majority of these diagnoses are cases of $\mathrm{PBC}$, other diagnoses, such as PSC or other causes of biliary cirrhosis, are likely to be included due to misclassification errors.

The study has the limitations inherent to all studies using administrative databases. In particular, the NIS: (1) only captures individual hospitalizations and not patients; (2) it neither enables the tracking of patients from one facility to 
another nor accounts for those with multiple hospitalizations within the same year; (3) it does not specify the cause of death; (4) it carries the risk of misclassification. Another possible concern derives from the use of a database aimed at hospitalized cases for the study of a slowly progressive disease such as PBC, when most patients are now cared for as outpatients.

Health disparities can occur due to differences between groups among many determinants, ranging from genetics to social environment, access to care, socioeconomic status, and education. The study of Adejumo et al. is relevant as it highlights differences in health access consistent with a wide body of research demonstrating that US racial and ethnic minorities are less likely to receive preventive medical treatments than Whites and often receive lower-quality care $[8,9]$, likely due to the lack of a universal, publicly funded healthcare system. Health disparities have been described for diverse liver diseases [10], including viral hepatitis, nonalcoholic liver disease, and hepatocellular carcinoma.

Health disparities among several groups have received increasing attention over the last decades; solving this problem has become a major public health priority. In order to increase the understanding of health disparities, there are many determinants of health beyond access to and provision of health care. Developing an integrated, multidisciplinary partnership between scientists, healthcare workers, public health experts, policymakers, payers, and patients is an effective approach to the complex challenge of identifying, understanding, and reducing health disparities in liver disease. Prioritizing the measurement of health disparities within institutions and among providers; building partnerships to enable patients to participate in developing solutions, in particular to gain insights into the struggle patients face; and making racial equity a strategic political priority are all steps that should decrease the current inequities. These steps are all required in order to make the pursuit of health equity a common practice.

\section{References}

1. Adejumo, AC, et al. Gender and racial differences in hospitalizations for primary biliary cholangitis in the United States. Dig Dis Sci. (Epub ahead of print). https://doi.org/10.1007/s10620-02006402-3.

2. Lu M, Li J, Haller IV, et al. Factors associated with prevalence and treatment of primary biliary cholangitis in United States Health Systems. Clin Gastroenterol Hepatol. 2018;16:1333-1341.e1336.

3. de Boer YS, Gerussi A, van den Brand FF, et al. Association between black race and presentation and liver-related outcomes of patients with autoimmune hepatitis. Clin Gastroenterol Hepatol. 2019;17:1616-1624.e1612.

4. Levy C, Naik J, Giordano C, et al. Hispanics with primary biliary cirrhosis are more likely to have features of autoimmune hepatitis and reduced response to ursodeoxycholic acid than non-Hispanics. Clin Gastroenterol Hepatol. 2014;12:1398-1405.

5. Tsou YK, Yeh CT. Primary biliary cirrhosis in antimitochondrial antibody-negative patients: Chang Gung Memorial Hospital experience. Chang Gung Med J. 2003;26:323-329.

6. Manno V, Gerussi A, Carbone M, et al. A national hospital-based study of hospitalized patients with primary biliary cholangitis. Hepatol Commun. 2019;3:1250-1257.

7. Carbone M, Mells GF, Pells G, et al. Sex and age are determinants of the clinical phenotype of primary biliary cirrhosis and response to ursodeoxycholic acid. Gastroenterology. 2013;144:560-569.

8. Orsi JM, Margellos-Anast H, Whitman S. Black-White health disparities in the United States and Chicago: a 15-year progress analysis. Am J Public Health. 2010;100:349-356.

9. Wyatt RLM, Botwinick L, Mate K, Whittington J. Achieving health equity: a guide for health care organizations. In: IHI White Paper; 2016.

10. Flores YN, Yee HF, Leng M, et al. Risk factors for chronic liver disease in Blacks, Mexican Americans, and Whites in the United States: results from NHANES IV, 1999-2004. Am J Gastroenterol. 2008;103:2231-2238.

Publisher's Note Springer Nature remains neutral with regard to jurisdictional claims in published maps and institutional affiliations. 